K.K. Shulembayeva*, Zh.Zh. Chunetova, S.B. Dauletbayeva, A.A. Tokubayeva, N.Zh. Omirbekova, Zh.K. Zhunusbayeva, A.I. Zhussupova

Al-Farabi Kazakh National University, Almaty, Kazakhstan

*E-mail: Kulziya.Shulembaeva@kaznu.kz

\title{
Some results of the breeding and genetic studies of common wheat in the south-east of Kazakhstan
}

\begin{abstract}
Increase productivity and quality of wheat by improving its genotype - one of the main problems of agriculture. Currently, using conventional methods of breeding and genetic research, such as mutagenesis, backcrossing selection and distant hybridization can increase the effectiveness of producing genetically improved crop forms. The fertility rate of soft wheat hybrids of wild species with T. timopheevi depends on the direction of crosses and genotype of varieties. Wild species as the parent component favorably affect a high percentage of tying grains $[1,2]$.

Key words: selection, chemical mutagenesis, isogenic lines, replacement of chromosomes, distant hybridization, variety, wheat chromosomes.
\end{abstract}

\section{Introduction}

By chemical mutagenesis new forms are obtained qualitatively, such as, dwarf mutants in wheat and barley, ultra-fast mutants in wheat and barley resistant to fungal diseases of plants form, high-lysine and highly productive mutants [3]. These facts indicate that mutants obtained by using chemical mutagens can successfully serve as progenitors of new high-yielding varieties. However, obtaining mutants and their study - is only the first stage of selection work. It is possible to use hybridization in selection of mutations. More important is the use of mutants in hybridization to obtain positive transgressions [4-8]. Preparation of mutants and their use for hybridization requires the study of genetic nature of emerging changes, which is crucial for the selection of effective mutagens with specific action, and to broaden and deepen understanding of the nature of wheat evolution. Mutants having complex morphological, physiological and biochemical changes affecting economically valuable properties can be further used to locate genes that determine the trait followed intervarietal replacement of chromosomes.

Isogenic lines are convenient objects for many biological and agricultural experiments.
Main advantage of these lines is high genetic similarity among themselves and with the control line, which allows estimating the contribution to the formation of crop marking characteristics and applying them as effective donor marker signs $[1,2]$.

One of the possibilities to create new varieties with economically valuable traits, and primarily in the direction of selection on productivity and disease resistance to wheat rust, and their improvement in economically valuable attributes is the method of hybridization. Interspecific hybridization in wheat breeding to leaf rust resistance requires use of $T$. timopheevii [3]. To eliminate the effect of hybrids sterility, to date, methods have been developed to facilitate the transfer of genes from distant species of wheat. Some of them are based on the methods of chromosome engineering, the other - on the methods of genetic control of meiotic recombination, and others - on the methods of genetic engineering. In this regard, research of our laboratory is focused on the use of a range of methods for selection of important initial forms of wheat.

The aim of our study is to obtain valuable mutant, isogenic lines with introgressive and economically valuable traits and their breeding and genetic analysis. 


\section{Materials and methods}

The object of research were $M_{1}, M_{2}, M_{3}$ mutants, obtained by $\mathrm{CdCl}_{2}$ processing of 4 varieties of soft wheat of local selection - Shagala, Kazakhstanskaya 3, Zhenis, Lutescens 32. Subsequently changed plants were noted as L-1 and L-2, Kazakhstanskaya 126 soft wheat variety (Triticum aestivum L. var. ferrugineum, $\mathrm{Al}$ ) and its analogues with marker genes that determine the number of morphological traits: $R h t$ - stunt disease of wheat, $E g$ - extended glumes, $B g$ - black coloring ear, $H g$ - pubescence ear, $C$ squareheadity of ear, $W$ - dewaxity, $B$ - short pitch tooth, $H p$ - pubescence of ear, $P c$ - purple straw, $R a$ - anthocyanin color of lobes, $P p$ - violet color pericarp grain genes $R a$ and $H l$, controlling the cilia on the ears and sinuses sheet densely pubescent leaf blade. Also, morphologically marked is isogenic line Saratovskaya 29. During the hybridization wild species of T. timopheevii, Nadezhda and Lutescens 32 varieties were used. In the experiment the following methods: mutagenesis, cytology, genetics, as well as hybridological, morphometric and statistical analyzes were applied.

Work to create isogenic lines of Kazakhstanskaya 126 variety was conducted by the standard technique recommended by Briggs and Knowles [5].

During hybridization, castration was performed by N.L. Udol'skaya technique [6], pollination by twirl-method [7].

Cytological studies were carried out at press time preparations with a microscope LOMO Mikmed-1.

Genetic analysis of $F_{1}$ and $F_{2}$ hybrids was carried out by qualitative and quantitative traits of wheat. Mathematical processing of data is reduced to finding the arithmetic mean and its error for the analyzed quantitative traits and to determine the reliability of the difference between the arithmetic means using Student's test (t) [9-11]. Accounting for chromosomal abnormalities in MI, AI and AII meiosis was performed on a temporary carmine preparations under the microscope MBI-3. The representativeness of the study results provide enough sample size $-60-100$ plants.

\section{Results and their discussion}

Cytological analysis of $M_{2}$ mutants. Chemical mutagens due to its ability to induce a high mutation rate are used in many countries around the world to create a breeding material. Chromosomal aberrations and violations of cell division is one of the main tests for mutagenicity of various influences.
The most revealing in this respect is the meiotic cell division, especially in objects such as wheat, with a large number of difficult identifiable chromosomes. Furthermore, violations, reaching the meiotic division, are more likely to be transmitted to the next generation.

In $\mathrm{M}_{1}$ generation of mutant plants percentage of broken cells was 35 , and 20 at anaphase AI and A II, indicating a significant decrease in the percentage of disorders in mutant $\mathrm{M}_{1}$ plants $(64 \%$ and $68 \%$ AI - A II). Violations in the form of phenomena of cytomixis (Figures 1-2) at $M_{1}$ counted $20-30 \%$ of the cells studied, while $M_{2}$ percentage of such cells decreased to $7-9 \%$. Thus, the percentage of mutant forms with disorders in $\mathrm{M}_{2}$ of Kazakhstanskaya 3 variety was $55 \%$, in contrast with disroders marked in $\mathrm{M}_{1}-90-95 \%$.

A similar decrease in the percentage of violations were observed in mutant varieties Zhenis, Lutescens 32 and Shagala. In AI and AII, and in tetrads minor violations as a lagging pole chromosome fragments, bridges, asynchronous division, and, occasionally, cells with no content are observed.

Cytological analysis of $M_{3}$ mutant plants. To characterize meiosis in mutant lines $\mathrm{M}_{3}$ and identification of monosomic, disomic plants in $\mathrm{F}_{1}$ hybrids with the mutant L-1 1080 cells were viewed.

Results of cytological analysis of $\mathrm{M}_{3}$ mutant plants showed that the percentage of cells with pyknosis in $\mathrm{M}_{3}$ mutants line L-1 of Kazakhstanskaya 3 variety was 0.29 ; mutant varieties Zhenis -0.10 ; Lutescens $32-0.23$; Shagala variety L-3 line -0.21 compared with $\mathrm{M}_{1}$ impaired cell (respectively). The proportion of cells with univalent were: $0.19 ; 0.009$; 0.16 (respectively).

Typical violations for mutant $\mathrm{M}_{1}-\mathrm{M}_{3}$ progeny plants were pyknosis; offset spindle of metaphase I; the presence of micronuclei univalents, polyvalents in tetrada; asynchronous cell division (Figures 3, 4).

This suggests that the changes occurred in the nuclear unit as a result of exposure to chemicals passed on from generation to generation. This fact testifies to the mutagenic effects used in our experiments with chemical compounds.

Thus, we have selected three specific mutant lines L-1, L-2 and L-3, modified on the basis of varieties Kazakhstanskaya 3 and Shagala. These lines are characterized by elongation of the ear and glume, large glassy grain (Figure 5) and the increased weight of 1000 grains. These important features selection and mutant forms, firmly inherited from generation to generation $\left(\mathrm{M}_{1}-\mathrm{M}_{6}\right)$. They are valuable, original forms are used in the selection process. 

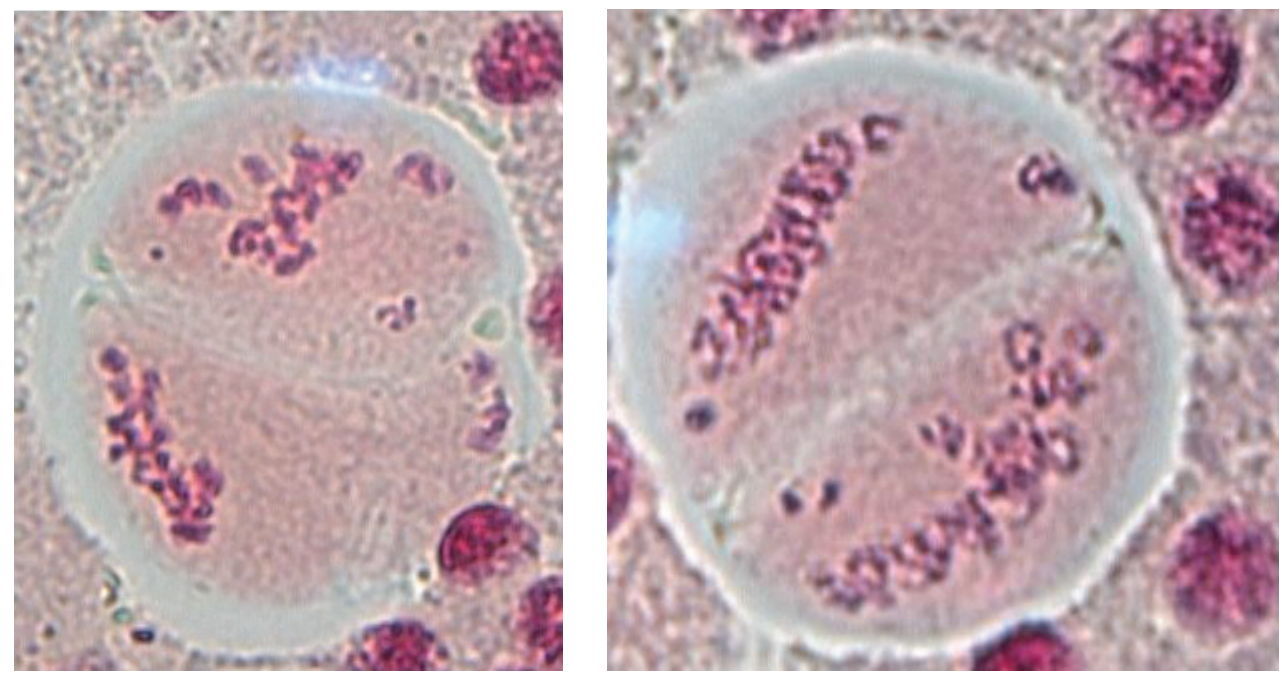

Figures 1, 2 - Cytomixis
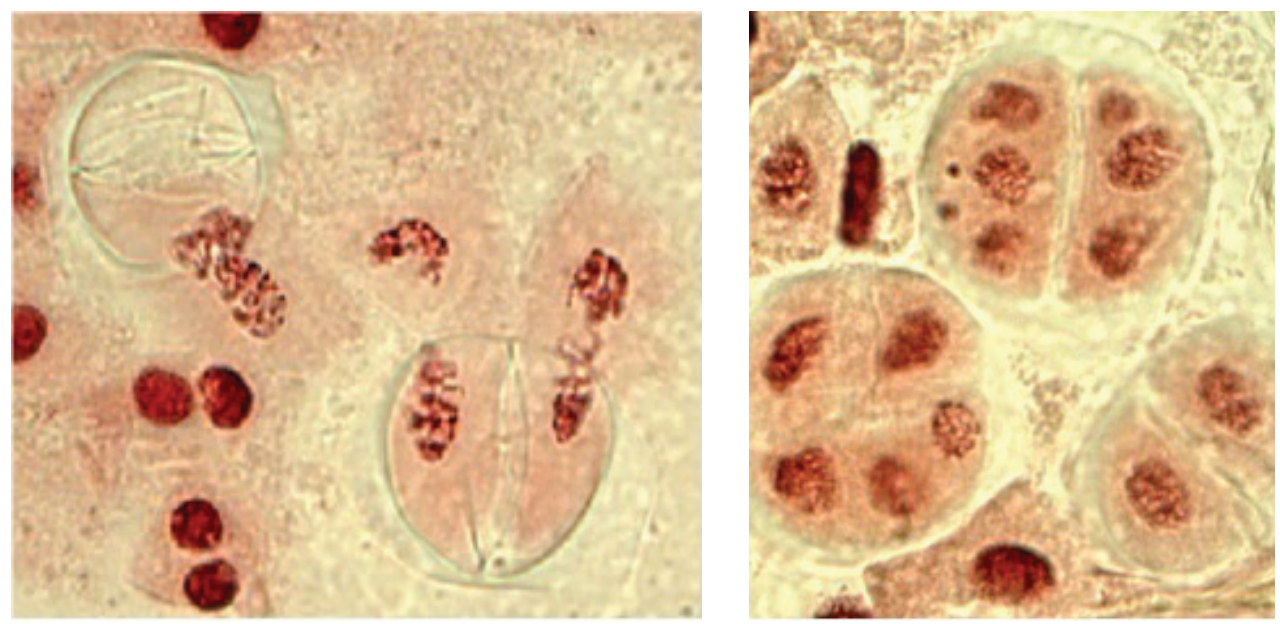

Figures 3, 4 - Cells without and with the micronuclei in tetrads

Marking of Kazakhstanskaya 126 variety for a number of morphological traits. For morphological marking of soft wheat Kazakhstanskaya 126 variety, isogenic line Saratovskaya 29 genes $\mathrm{Hg}, \mathrm{Bg}, \mathrm{C}, \mathrm{Pr}, \mathrm{Eg}, \mathrm{Hp}, \mathrm{B}, \mathrm{Rht}, \mathrm{Pc}, \mathrm{W}, \mathrm{Ra}$, $P a$, kindly provided to us by O.I. Maystrenko (ICG SB RAS, Novosibirsk) were used as the donor marker signs.

Backcross method according to F. Briggs and P. Knowles was used for creation of isogenic lines [5]. When transferring marker genes to the genotype of the recurrent variety of in the scheme of the complete continuous backcrossing all plants are $F_{1}$ hybrids were a sign of the introduced genes, which allowed to judge their dominant inheritance. The subsequent introduction of a marker gene was performed using the next offspring backcrosses to backcross plants recipient grade, as to recover the recurrent genotype varieties must hold at least six to eight saturating crossings.

During the backcrossing we conducted a careful selection of the desired genotypes of similar morphotype with the recurrent parent and differing on the basis of the marker. Considering the period of display marking trait in hybrids, the amount taken for crossing plants was regulated. Isogenic lines of Kazakhstanskaya 126 variety signs - anthocyanin color of lobes (gene $R a$ ), the presence of cilia in the ears sheet sinus $(\mathrm{Pa})$, pubescence of the ear $(\mathrm{Hp})$, dewaxity $(W)$, shortness $(R h t)$, violet color of the anthers (Pan), pubescent leaves $(H l)$, are tested before flowering. In this case, the crossing with only 1-2 ears was used. 
In case of late manifestations marking signs, such as the black coloring of the ear $(\mathrm{Bg})$, glume pubescence $(\mathrm{Hg})$, club-shaped spike (C), violet color grain (PP), short pitch tooth (B), in the crossing of isogenic lines of all the plants involved.

The annual search for signs of marker allows a series of isogenic lines complement other morphological marker signs. Marker sign of the presence of cilia in the ears sheet sinus is well tested and most fully manifested during booting. At the stage of wax and milk ripeness signs are less pronounced. On the basis of pubescent leaves used as a donor line with the clutch 3 characteristics: pubescence of the leaf blade and the ear, the presence of anthocyanin ears. All the signs were the dominant mode of inheritance. In the selection of the desired genotypes in fissile generations of hybrid $\mathrm{F}_{1}$ plants, we were able to select plants are phenotypically similar to the recurrent parent with inherited sign of interest - pubescent leaves. According to the lines backcrossing is being made with careful selection of plants on the marker.

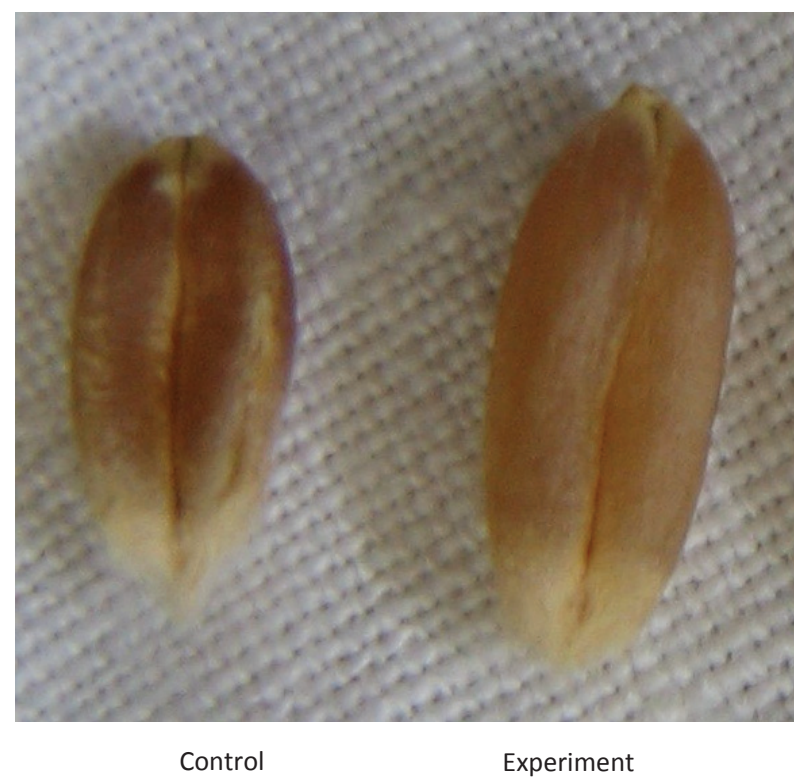

Figure 5 - Control and experimental grain

With regard to manifestations of anthocyanin pigmentation in different plant organs, interesting morphological markers may serve as a sign of violet color of the anthers, which manifests itself in the flowering stage of wheat. As a donor of this feature can serve as a mutant line L-1 of our collection.

Structural analysis of elements of efficiency isogenic lines revealed three lines - IL-Hg, IL-BgHg and IL-Pc, which differ significantly exceeding the target spike productivity and weight of 1000 grains in comparison with control of Kazakhstanskaya 126 variety and other lines. The increase in the average weight of grain from these isogenic lines confirmed the improvement of grain filling. This is probably due to the increase in productivity of photosynthesis due to the intensification of plant anthocyanin.

Interspecific hybridization. Tetraploid endemic species Triticum timopheevii Zhuk. (genomic formula $A^{t} A^{t} G G$ ) is characterized by a unique pool of genes controlling resistance to a large number of wheat diseases. Creating and intensive involvement in the selection process of soft wheat donors with effective Lr-resistance genes transmitted from wild relatives, could significantly expand its genetic basis for this or other economically valuable traits.

Available data on the compatibility of $T$. aestivum and T. timopheevii types is inconsistent.

In various soil and climatic conditions in Russia and abroad varied material source of parent species has been involved in the hybridization. Contradictory results are explained as genetic features of parent forms and originality of specific ecological zones $[3,4]$.

Shown in Table 1 experimental data suggests that crossing wheat with different kinds of wild crops were successful. However, tying the grains in various combinations ranging from 0 to $64.18 \%$. Apparently, the percentage of success depends on 
the genotype of accessions taken for crossing, as well as the direction of the crossing.

Thus, the percentage of successful result of crossing $T$. timopheevii with soft wheat is relatively high in the case where the shape is taken as the parent wild form.

Depending on the number of pollinated ears the tying of hybrid progeny grains were different. Level of compatibility with T. timopheevii wheat variety named Nadezhda is relatively high, and the average is about $62.63 \%, \mathrm{~K}-2780-40.67 \%$, and the percentage of luck backcross hybrid offspring plummets to $15.28 \%$ and $10 \%$, respectively .

$\mathrm{F}_{1}$ hybrids (T. timopheevi to $\left.\mathrm{x}-2780\right) 150-61 \%$, and in the reciprocal crosses of 56 pollinated flowers ensued only $10 \%$ of the grain.
Thus, the study of reciprocal hybrids $\mathrm{F}_{1}$, derived from crosses of soft wheat with wild view $-T$. timopheevi clear differences were found in the percentage tying grains. In plants, a hybrid combination obtained by crossing T. timopheevi with soft wheat, that is where as the female was used soft wheat, the percentage of luck somewhat lower than hybrids, where as the parent form serves wild species.

Results obtained showed that the use of wild species with reciprocal crosses it with soft wheat as a parent component improves compatibility genomes. Hybrids derived from crosses of T. timopheevi with soft wheat have characteristic heteroplasmic state: while there are copies of the wild (maternal) and wheat (paternal) types.

Table 1 - Fertility of reciprocal hybrids hybridization

\begin{tabular}{|c|c|c|c|c|}
\hline \multirow{2}{*}{ No. } & \multirow{2}{*}{ Combination of cross-breeding } & \multicolumn{2}{|c|}{ Number } & \multirow{2}{*}{ Percent, tying grains $\%$} \\
\hline & & of pollinated flowers & of knotted grains & \\
\hline & \multicolumn{4}{|c|}{ Soft wheat $x$ T.timopheevi } \\
\hline 1 & $\mathrm{~F}_{0}$ (T.timopheevi $\mathrm{x}$ Nadezhda) & 190 & 119 & 62,63 \\
\hline 2 & $\mathrm{~F}_{0}$ (Hope $\mathrm{x}$ T.timopheevi) & 72 & 11 & 15,28 \\
\hline 3 & $\mathrm{~F}_{0}($ T.timopheevi х к-2780) & 150 & 61 & 40,67 \\
\hline 4 & $\mathrm{~F}_{0}(\kappa-2780 \times$ T.timopheevi $)$ & 56 & 6 & 10 \\
\hline 5 & $\mathrm{~F}_{0}(32$ short. x T.timopheevi $)$ & 56 & 0 & 0 \\
\hline
\end{tabular}

\section{References}

1. Krupnov V.A. About the establishment of isogenic lines of hard and soft wheat, adapted to the conditions of the Volga region // the Agricultural biology. Ser. Plant Biology. - 1995. - No. 5. - P. 31-37.

2. Koval S.F. Study of variety models of spring wheat in the isogenic lines and analogues. Problems and prospects // Problems of breeding crops Novosibirsk ICG SB RAS, 2002. - P. 56-68.

3. Timonova E.M., Leonova I.N., Belan I.A., Rosseeva L.P., Salina E.A. The influence of individual sections of chromosomes Triticum timopheevii building resilience to disease and quantitative traits
// Vavilov Journal of Genetics and Breeding. - 2012. - Vol. 16, No. 1. - P. 142-159.

4. Aksyonova E., Sinyavskaya M., Danilenko N. et al. Heteroplasmy and paternally oriented shift of the organellar DNA composition in barley-wheat hybrids during backcrosses with wheat parents // Genome. - 2005. - Vol. 48. - P. 761-769.

5. Briggs F., Knowles P. Scientific basis of plant breeding. - M .: Kolos. 1972. -399 p.

6. Udol'skaya N.L. Selection of spring wheat. Almaty, 1961. - 183 pp.

7. Borlaug N.E. Wheat breeding and its impact on world food supply // Wheat Genet. Symp. - 1980. - P. 36. 